\title{
Projective reduce order synchronization of fractional order chaotic systems with unknown parameters
}

\author{
M. Mossa Al-sawalha \\ Mathematics Department, Faculty of Science, University of Hail, Kingdom of Saudi Arabia.
}

Communicated by D. Baleanu

\begin{abstract}
This paper, mainly concerns the adaptive projective reduce order synchronization behavior of uncertain chaotic system. By Lyapunov stability theory, the adaptive control law and the parameter update law are derived to make the states of two chaotic and hyperchaotic systems asymptotically synchronized up to a desired identical and different scaling matrix. Numerical simulation results show that the proposed method is effective, convenient, and also faster for projective dual synchronization of chaotic and hyperchaotic systems. (C)2017 All rights reserved.
\end{abstract}

Keywords: Projective, reduce order synchronization, adaptive control, unknown parameters, Lyapunov stability theory. 2010 MSC: 3C10, 93C95.

\section{Introduction}

The applications of fractional order chaotic and hyperchaotic systems are bountiful all around us and its interest is fast gaining momentum. The science of fractional chaos is an interdisciplinary field which crosses boundaries in the academic world such as economics, engineering, biology, chemistry, physics, and etc. Issues in topics such as, the synchronization of order chaotic systems in a broad variety of situations and the use of fractional order chaotic dynamics for various purposes, are at the forefront of recent application topics in nonlinear science. These topics unites the knowledge of basic mathematical properties of fractional order chaos and specific practical considerations of various applications $[7,15,19,26,27,31,32]$. Synchronization of chaotic system with integer order is understood well since the pioneering work by Pecora and Carrol [18]. Studies on chaos synchronization for the fractional order systems are just beginning to attract some attention due to its potential applications in secure communication and control processing. There are many different methods and strategies of fractional order continuous and discrete chaos synchronization have been developed such as activation feedback method, linear and nonlinear feedback synchronization $[3,4,8,13,20,25,29]$, sliding mode control [12, 16, 22, 23, 30, 34], adaptive control [1, 2, 5, 9, 14, 21, 33], and projective synchronization [10, 17, 24]. To the best of our knowledge, most of research efforts mentioned above have concentrated on studying the synchronization

Email address: sawalha_moh@yahoo.com (M. Mossa Al-sawalha)

doi:10.22436/jnsa.010.04.64

Received 2017-02-12 
of fractional order chaotic systems whose models are identical, different. However, synchronization of fractional order chaotic systems also can be induced even in strictly different order or reduced order synchronization [6], especially the systems in biological science and social science. So, the study of reduced order synchronization is very important from the perspective of control theory and very necessary from the perspective of practical application. Reduced order synchronization is the problem of synchronizing a slave system with projections of a master system. In this paper, we will show that the projective reduced order synchronization between different fractional order chaotic systems may be used to manipulate the scaling factor such that the drive and response systems could be synchronized up to a desired scaling matrix, on the other hand, since the fractional order chaotic systems have uncertain parameters, it is difficult to find the projective reduced order synchronization. In this case, modified adaptive control is an effective method for synchronizing the fractional order chaotic systems with uncertain parameters in the sense of projective reduced order synchronization.

The rest of the paper is organized as follows. In Section 2, we briefly describe the problem. In Sections 3 and 4 we describe adaptive projective reduced-order synchronization strategies in different projection with parameter update law for fourth order fractional order hyperchaotic Chen system and third order fractional order Genesio-Tesi system. Conclusions are given in Section 5.

\section{Problem description and control design for the projective reduce order synchronization}

\subsection{Problem description}

Given the fractional order chaotic system, i.e., the drive system is

$$
D_{t}^{q} x=f(x)+F(x) \alpha,
$$

where $x=\left(x_{1}, x_{2}, \ldots, x_{m}\right)^{\top} \in R^{m}$ is the state vector of the system, $\alpha \in R^{k}$ is the unknown parameter vector of the system, and $f(x): R^{m} \rightarrow R^{m}, F(x): R^{m} \rightarrow R^{m \times k}$. On the other hand, the controlled response system is given by:

$$
D_{t}^{q} y=g(y)+G(y) \beta+u,
$$

where $y=\left(y_{1}, y_{2}, \ldots, y_{n}\right)^{\top} \in R^{n}$ is the state vector of the system, $\beta \in R^{\ell}$ is the unknown parameter vector of the system, and $g(y): R^{n} \rightarrow R^{n}, G(y): R^{n} \rightarrow R^{n \times l}, U \in R^{n}$. When order $n=m, l=k$ and the functions $f=g, F=G$, the response system is identical to the drive system, and the projective synchronization problem has been well studied $[10,17,24]$. When two systems satisfy the condition $m<n$ (of course $f \neq g$, and $F \neq G$ ), that is, the order of the response system is lower than that of the drive system, the projective synchronization is only attained in reduce-order. Therefore, we can divide the master system into two parts. The projection:

$$
D_{t}^{q} x_{i}=f_{i}(x)+F_{i}(x) \alpha
$$

where $x_{i} \in R^{n}, f_{i}: R^{m} \rightarrow R^{n}$, and $F_{i}: R^{m} \rightarrow R^{n \times k}$, and the rest:

$$
D_{t}^{q} x_{j}=f_{j}(x)+F_{j}(x) \alpha,
$$

where $x_{j} \in R^{u}, f_{j}: R^{m} \rightarrow R^{u}$, and $F_{j}: R^{m} \rightarrow R^{u \times k}$, and orders $n, u$ satisfy $n+u=m$. The purpose of the reduce order synchronization is to design a controller $\mathrm{U}$, which is able to synchronize the two identical or different chaotic (hyper-chaotic) systems with a scaling matrix, i.e.,

$$
\lim _{t \rightarrow \infty}\left\|y(t)-H x_{i}(t)\right\|=0,
$$

where $\mathrm{H}$ is called the scaling matrix and $\|\cdot\|$ represents the Euclidean norm. 
Lemma 2.1 ([1, 2, 19]). If $p>q \geqslant 0, m$ and $n$ are integers such that $0 \leqslant m-1 \leqslant p<m, 0 \leqslant n-1 \leqslant q<n$, then we obtain

$$
{ }_{a} D_{t}^{q}\left({ }_{a} D_{t}^{-q} f(t)\right)={ }_{a} D_{t}^{p-q} f(t) .
$$

Lemma $2.2([1,2,19])$. If $p, q \geqslant 0$, then there exist integers $m$ and $n$ such that $0 \leqslant m-1 \leqslant p<m, 0 \leqslant n-1 \leqslant$ $\mathrm{q}<\mathrm{n}$, then

$$
{ }_{a} D_{t}^{p}\left({ }_{a} D_{t}^{q} f(t)\right)={ }_{a} D_{t}^{p+q} f(t)-\sum_{j=1}^{n}\left[{ }_{a} D_{t}^{q-j} f(t)\right]_{t=a} \frac{(t-a)^{-p-j}}{\Gamma(1-p-j)} .
$$

Lemma $2.3([1,2,19])$. Suppose $f(t)$ has a continuous $k$ th derivative in $[0, t](k \in N, t>0)$ and let $p, q>0$, then there exists some $n \in N$ with $n \leqslant k$ and $p, p+q \in[n-1, n]$, then

$$
{ }_{a} D_{t}^{q}{ }_{a} D_{t}^{q} f(t)={ }_{a} D_{t}^{p+q} f(t)
$$

\subsection{Projective reduce order synchronization controller design}

The error vector between the systems (2.1) and (2.2) can be expressed as

$$
D_{t}^{q} e(t)=g(y)+G(y) \beta-H f_{i}(x)-H F_{i}(x) \alpha+U .
$$

Here, let the control function $\mathrm{U}$ be

$$
\begin{aligned}
\mathrm{U}= & H f_{i}(x)+H F_{i}(x) \alpha-g(y)-G(y) \beta \\
& +D_{t}^{q-1}\left[H F_{i}(x) \hat{\alpha}-G(y) \hat{\beta}-\left(D_{t}^{q-1} e(t)\right) \frac{(t)^{-(q-1)-1}}{\Gamma(-(q-1))}-e\right]
\end{aligned}
$$

and adaptive laws of parameters are taken as

$$
\dot{\hat{\alpha}}=-\left[\mathrm{F}_{\mathrm{i}}(\mathrm{x})\right]^{\mathrm{T}} \mathrm{He}, \quad \dot{\hat{\beta}}=[\mathrm{G}(\mathrm{y})]^{\mathrm{T}} e,
$$

where, $\hat{\alpha}, \hat{\beta}$ are estimated values of $\alpha, \beta$, respectively.

Theorem 2.4. For given synchronization scaling matrix $\mathrm{H}=\operatorname{diag}\left(\mathrm{h}_{1}, \mathrm{~h}_{2}, \ldots, \mathrm{h}_{\mathrm{n}}\right)$ and any initial conditions, projective reduce order synchronization between system (2.1) and system (2.2) will occur by the control law (2.4) and the updating law of parameters (2.5).

Proof. Inserting (2.4) into (2.3) yields the following:

$$
D_{t}^{q} e(t)=D_{t}^{q-1}\left[H F_{i}(x)(\hat{\alpha}-\alpha)-G(y)(\hat{\beta}-\beta)-\left(D_{t}^{q-1} e(t)\right) \frac{(t)^{-(q-1)-1}}{\Gamma(-(q-1))}-e\right] .
$$

Construct a positive Lyapunov function candidate in the form of:

$$
V=\frac{1}{2}\left[e^{\top} e+\tilde{\alpha}^{\top} \tilde{\alpha}+\tilde{\beta}^{\top} \tilde{\beta}\right]
$$

where $\tilde{\alpha}=\hat{\alpha}-\alpha, \tilde{\beta}=\hat{\beta}-\beta$. The time derivative of $V$ along the trajectory of the error dynamical system (2.6) is as follows

$$
\dot{V}=\left[e^{\top} \dot{e}+\dot{\tilde{\alpha}}^{\top} \tilde{\alpha}+\dot{\tilde{\beta}}^{\top} \tilde{\beta}\right]
$$


Using Lemma 2.2 in Eq. (2.7) we get

$$
\dot{V}=e^{T}\left[D_{t}^{q-1}\left(D_{t}^{q} e(t)\right)+\left(D_{t}^{q-1} e(t)\right) \frac{(t)^{-(q-1)-1}}{\Gamma(-(q-1))}\right]+\dot{\tilde{\alpha}}^{\top} \tilde{\alpha}+\dot{\tilde{\beta}}^{\top} \tilde{\beta}
$$

From Eqs. (2.5) and (2.7), we get

$$
\begin{aligned}
\dot{V}= & e^{\top}\left[D_{t}^{q-1}\left(D_{t}^{q-1}\left[H F_{i}(x) \tilde{\alpha}-G(y) \tilde{\beta}-\left(D_{t}^{q-1} e(t)\right) \frac{(t)^{-(q-1)-1}}{\Gamma(-(q-1))}-e\right]\right)\right. \\
& \left.+\left(D_{t}^{q-1} e(t)\right) \frac{(t)^{-(q-1)-1}}{\Gamma(-(q-1))}\right]+\dot{\tilde{\alpha}}^{\top} \tilde{\alpha}+\dot{\tilde{\beta}}^{\top} \tilde{\beta} .
\end{aligned}
$$

Now, using Lemma 2.1 and Eq. (2.5), Eq. (2.8) reduces to

$$
\begin{aligned}
\dot{V}= & e^{\top}\left[H F(x) \tilde{\alpha}-G(y) \tilde{\beta}-\left(D_{t}^{q-1} e(t)\right) \frac{(t)^{-(q-1)-1}}{\Gamma(-(q-1))}-e\right. \\
& \left.+\left(D_{t}^{q-1} e(t)\right) \frac{(t)^{-(q-1)-1}}{\Gamma(-(q-1))}\right]-e^{\top} H F(x) \tilde{\alpha}+e^{\top} G(y) \tilde{\beta}=-e^{\top} e<0 .
\end{aligned}
$$

According to the Lyapunov stability theory, the error variable becomes zero as time $t$ tends to infinity, i.e., $\lim _{t \rightarrow \infty}\|e(t)\|=0$. This means that the drive system (2.1) and the response system (2.2) achieved the projective reduce order synchronization about the scaling matrix $\mathrm{H}$. This completes the proof.

\section{Projective reduce order synchronization between the projection $(x y z)$ of the fractional-order hyper- chaotic Chen and Genesio-Tesi systems}

In this section, we take the projection (xyz) of the fractional-order hyperchaotic Chen system [28] and Genesio-Tesi system [11] into consideration. The fractional-order hyperchaotic Chen system is described as follows:

$$
\begin{aligned}
D_{t}^{q_{1}} x_{1} & =a_{1}\left(y_{1}-x_{1}\right)+w_{1}, \\
D_{t}^{q_{2}} y_{1} & =d_{1} x_{1}-x_{1} z_{1}+c_{1} y_{1}, \\
D_{t}^{q_{3}} z_{1} & =x_{1} y_{1}-b_{1} z_{1}, \\
D_{t}^{q_{4}} w_{1} & =y_{1} z_{1}+r_{1} w_{1},
\end{aligned}
$$

and the fractional-order Genesio-Tesi system is described as follows:

$$
\begin{aligned}
& D_{t}^{\mathrm{q}_{1}} x_{2}=y_{2}+u_{1}, \\
& D_{t}^{\mathrm{q}_{2}} y_{2}=z_{2}+u_{2}, \\
& D_{t}^{\mathrm{q}_{3}} z_{2}=-a_{2} x_{2}-b_{2} y_{2}-c_{2} z_{2}+x_{2}^{2}+u_{3},
\end{aligned}
$$

where $q$ is the fractional-order satisfying $0<q \leqslant 1$ and $u_{1}, u_{2}, u_{3}$ are three control functions to be designed and all parameters $a_{1}, d_{1}, c_{1}, b_{1}, r_{1}, a_{2}, b_{2}, c_{2}$ are unknown. The error system between system (3.1) and system (3.2) can be defined as

$$
\begin{aligned}
& D_{t}^{q_{1}} e_{1}(t)=\left(e_{2}+h_{2} y_{1}\right)-h_{1} a_{1}\left(y_{1}-x_{1}\right)-h_{1} w_{1}+u_{1}, \\
& D_{t}^{q_{2}} e_{2}(t)=\left(e_{3}+h_{3} z_{1}\right)-h_{2} d_{1} x_{1}+h_{2} x_{1} z_{1}-h_{2} c_{1} y_{1}+u_{2}, \\
& D_{t}^{q_{3}} e_{3}(t)=-a_{2}\left(e_{1}+h_{1} x_{1}\right)-b_{2}\left(e_{2}+h_{2} y_{1}\right)-c_{2}\left(e_{3}+h_{3} z_{1}\right)+\left(e_{1}+h_{1} x_{1}\right)^{2}-h_{3} x_{1} y_{1}+h_{3} b_{1} z_{1}+u_{3},
\end{aligned}
$$


where $e_{1}=x_{2}-h_{1} x_{1}, e_{2}=y_{2}-h_{2} y_{1}, e_{3}=z_{2}-h_{3} z_{1}$ and $h=\operatorname{dig}\left(h_{1}, h_{2}, h_{3}\right)$ is the scaling matrix. Our goal is to derive the controller $\mathrm{U}$ with a parameter estimation update law such that Eqs. (3.1) synchronize Eqs. (3.2), the controllers are taken as

$$
\begin{aligned}
u_{1}= & -\left(e_{2}+h_{2} y_{1}\right)+h_{1} a_{1}\left(y_{1}-x_{1}\right)+h_{1} w_{1}+D_{t}^{q_{1}-1}\left[h_{1} \hat{a}_{1}\left(y_{1}-x_{1}\right)\right. \\
& \left.-\left(D_{t}^{q_{1}-1} e_{1}(t)\right) \frac{(t)^{-\left(q_{1}-1\right)-1}}{\Gamma\left(-\left(q_{1}-1\right)\right)}-e_{1}\right], \\
u_{2}= & -\left(e_{3}+h_{3} z_{1}\right)+h_{2} d_{1} x_{1}-h_{2} x_{1} z_{1}+h_{2} c_{1} y_{1}+D_{t}^{q_{2}-1}\left[h_{1} \hat{d}_{1} x_{1}+h_{2} \hat{c}_{1} y_{1}\right. \\
& \left.-\left(D_{t}^{q_{2}-1} e_{2}(t)\right) \frac{(t)^{-\left(q_{2}-1\right)-1}}{\Gamma\left(-\left(q_{2}-1\right)\right)}-e_{2}\right], \\
u_{3}= & a_{2}\left(e_{1}+h_{1} x_{1}\right)+b_{2}\left(e_{2}+h_{2} y_{1}\right)+c_{2}\left(e_{3}+h_{3} z_{1}\right)-\left(e_{1}+h_{1} x_{1}\right)^{2}+h_{3} x_{1} y_{1} \\
& -h_{3} b_{1} z_{1}+D_{t}^{q_{3}-1}\left[\hat{a}_{2}\left(e_{1}+h_{2} x_{1}\right)+\hat{b}_{2}\left(e_{2}+h_{2} y_{1}\right)+\hat{c}_{2}\left(e_{3}+h_{3} z_{1}\right)-h_{3} \hat{b}_{1} z_{1}\right. \\
& \left.-\left(D_{t}^{q_{3}-1} e_{3}(t)\right) \frac{(t)^{-\left(q_{3}-1\right)-1}}{\Gamma\left(-\left(q_{3}-1\right)\right)}-e_{3}\right],
\end{aligned}
$$

and parameter update rules are

$$
\begin{aligned}
& \dot{\hat{\mathrm{a}}}_{1}=-\mathrm{h}_{1}\left(\mathrm{y}_{1}-\mathrm{x}_{1}\right) e_{1}, \\
& \dot{\hat{\mathrm{d}}}_{1}=-\mathrm{h}_{2} \mathrm{x}_{1} e_{2}, \\
& \dot{\hat{\mathrm{c}}}_{1}=-\mathrm{h}_{2} \mathrm{y}_{1} \mathrm{e}_{2}, \\
& \dot{\hat{\mathrm{b}}}_{1}=\mathrm{h}_{3} z_{1} e_{3}, \\
& \dot{\hat{\mathrm{a}}}_{2}=-\left(e_{1}+\mathrm{h}_{1} x_{1}\right) e_{3}, \\
& \dot{\hat{\mathrm{b}}}_{2}=-\left(e_{2}+\mathrm{h}_{2} \mathrm{y}_{1}\right) e_{3}, \\
& \dot{\hat{\mathrm{c}}}_{2}=-\left(e_{3}+\mathrm{h}_{3} z_{1}\right) e_{3},
\end{aligned}
$$

where, $\hat{a}_{1}, \hat{\mathrm{d}}_{1}, \hat{\mathrm{c}}_{1}, \hat{b}_{1}, \hat{a}_{2}, \hat{b}_{2}, \hat{c}_{2}$ are estimates of $\mathrm{a}_{1}, \mathrm{~d}_{1}, \mathrm{c}_{1}, \mathrm{~b}_{1}, \mathrm{a}_{2}, \mathrm{~b}_{2}, \mathrm{c}_{2}$, respectively.

Theorem 3.1. For given synchronization scaling matrix $\mathrm{h}=\operatorname{diag}\left(\mathrm{h}_{1}, \mathrm{~h}_{2}, \ldots, \mathrm{h}_{\mathrm{n}}\right)$ and any initial conditions, projective reduce order synchronization between system (3.1) and system (3.2) will occur by the control law (3.4) and the updating law of parameters (3.5).

Proof. Inserting (3.4) into (3.3) yields the following:

$$
\begin{aligned}
D_{t}^{q_{1}} e_{1}(t)= & D_{t}^{q_{1}-1}\left[h_{1} \tilde{a}_{1}\left(y_{1}-x_{1}\right)-\left(D_{t}^{q_{1}-1} e_{1}(t)\right) \frac{(t)^{-\left(q_{1}-1\right)-1}}{\Gamma\left(-\left(q_{1}-1\right)\right)}-e_{1}\right], \\
D_{t}^{q_{2}} e_{2}(t)= & D_{t}^{q_{2}-1}\left[h_{2} \tilde{d}_{1} x_{1}+h_{2} \tilde{c}_{1} y_{1}-\left(D_{t}^{q_{2}-1} e_{2}(t)\right) \frac{(t)^{-\left(q_{2}-1\right)-1}}{\Gamma\left(-\left(q_{2}-1\right)\right)}-e_{2}\right], \\
D_{t}^{q_{3}} e_{3}(t)= & D_{t}^{q_{3}-1}\left[\tilde{a}_{2}\left(e_{1}+h_{1} x_{1}\right)+\tilde{b}_{2}\left(e_{2}+h_{2} y_{1}\right)+\tilde{c}_{2}\left(e_{3}+h_{3} z_{1}\right)-h_{3} \tilde{b}_{1} z_{1}\right. \\
& \left.-\left(D_{t}^{q_{3}-1} e_{3}(t)\right) \frac{(t)^{-\left(q_{3}-1\right)-1}}{\Gamma\left(-\left(q_{3}-1\right)\right)}-e_{3}\right],
\end{aligned}
$$

where $\tilde{a}_{1}=\hat{a}_{1}-a_{1}, \tilde{d}_{1}=\hat{d}_{1}-d_{1}, \tilde{c}_{1}=\hat{c}_{1}-c_{1}, \tilde{b}_{1}=\hat{b}_{1}-b_{1}, \tilde{a}_{2}=\hat{a}_{2}-a_{2}, \tilde{b}_{2}=\hat{b}_{2}-b_{2}, \tilde{c}_{2}=\hat{c}_{2}-c_{2}$. Consider the following Lyapunov function candidate:

$$
\mathrm{V}=\frac{1}{2}\left(e^{\top} e+\tilde{\mathrm{a}}_{1}^{2}+\tilde{\mathrm{d}}_{1}^{2}+\tilde{\mathrm{c}}_{1}^{2}+\tilde{\mathrm{b}}_{1}^{2}+\tilde{\mathrm{a}}_{2}^{2}+\tilde{\mathrm{b}}_{2}^{2}+\tilde{\mathrm{c}}_{2}^{2}\right)
$$


The time derivative of $\mathrm{V}$ along the solution of error dynamical system equation (3.7) gives

$$
\dot{\mathrm{V}}=\left(e_{1} \dot{\mathrm{e}}_{1}+\mathrm{e}_{2} \dot{\mathrm{e}}_{2}+e_{3} \dot{\mathrm{e}}_{3}+\tilde{\mathrm{a}}_{1} \dot{\tilde{\mathrm{a}}}_{1}+\tilde{\mathrm{d}}_{1} \dot{\tilde{\mathrm{d}}}_{1}+\tilde{\mathrm{c}}_{1} \dot{\tilde{\mathrm{c}}}_{1}+\tilde{\mathrm{b}}_{1} \dot{\tilde{\mathrm{b}}}_{1}+\tilde{\mathrm{a}}_{2} \dot{\tilde{\mathrm{a}}}_{2}+\tilde{\mathrm{b}}_{2} \dot{\tilde{\mathrm{b}}}_{2}+\tilde{\mathrm{c}}_{2} \dot{\tilde{\mathrm{c}}}_{2}\right)
$$

Using Lemma 2.2 in Eq. (3.8) we get

$$
\begin{aligned}
& \dot{V}=\left(e_{1}\left[D_{t}^{1-q_{1}}\left(D_{t}^{q_{1}} e_{1}(t)\right)+\left(D_{t}^{q_{1}} e_{1}(t)\right) \frac{(t)^{-\left(q_{1}-1\right)-1}}{\Gamma\left(-\left(q_{1}-1\right)\right)}\right]\right. \\
& +e_{2}\left[D_{t}^{1-q_{2}}\left(D_{t}^{q_{2}} e_{2}(t)\right)+\left(D_{t}^{q_{2}} e_{2}(t)\right) \frac{(t)^{-\left(q_{2}-1\right)-1}}{\Gamma\left(-\left(q_{2}-1\right)\right)}\right] \\
& +e_{3}\left[D_{t}^{1-q_{3}}\left(D_{t}^{q_{3}} e_{3}(t)\right)+\left(D_{t}^{q_{3}} e_{3}(t)\right) \frac{(t)^{-\left(q_{3}-1\right)-1}}{\Gamma\left(-\left(q_{3}-1\right)\right)}\right] \\
& \left.+\tilde{\mathrm{a}}_{1} \dot{\tilde{\mathrm{a}}}_{1}+\tilde{\mathrm{d}}_{1} \dot{\tilde{\mathrm{d}}}_{1}+\tilde{\mathrm{c}}_{1} \dot{\tilde{\mathrm{c}}}_{1}+\tilde{\mathrm{b}}_{1} \dot{\tilde{\mathrm{b}}}_{1}+\tilde{\mathrm{a}}_{2} \dot{\tilde{\mathrm{a}}}_{2}+\tilde{\mathrm{b}}_{2} \dot{\tilde{\mathrm{b}}}_{2}+\tilde{\mathrm{c}}_{2} \dot{\tilde{\mathrm{c}}}_{2}\right), \\
& =\left(e _ { 1 } \left[D_{t}^{1-q_{1}}\left(D_{t}^{q_{1}-1}\left[h_{1} \tilde{a}_{1}\left(y_{1}-x_{1}\right)-\left(D_{t}^{q_{1}-1} e_{1}(t)\right) \frac{(t)^{-\left(q_{1}-1\right)-1}}{\Gamma\left(-\left(q_{1}-1\right)\right)}-e_{1}\right]\right)\right.\right. \\
& \left.+\left(D_{t}^{q_{1}} e_{1}(t)\right) \frac{(t)^{-\left(q_{1}-1\right)-1}}{\Gamma\left(-\left(q_{1}-1\right)\right)}\right] \\
& +e_{2}\left[D_{t}^{1-q_{2}}\left(D_{t}^{q_{2}-1}\left[h_{2} \tilde{d}_{1} x_{1}+h_{2} \tilde{c}_{1} y_{1}-\left(D_{t}^{q_{2}-1} e_{2}(t)\right) \frac{(t)^{-\left(q_{2}-1\right)-1}}{\Gamma\left(-\left(q_{2}-1\right)\right)}-e_{2}\right]\right)\right. \\
& \left.+\left(D_{t}^{q_{2}} e_{2}(t)\right) \frac{(t)^{-\left(q_{2}-1\right)-1}}{\Gamma\left(-\left(q_{2}-1\right)\right)}\right] \\
& +e_{3}\left[D _ { t } ^ { 1 - q _ { 3 } } \left(D _ { t } ^ { 1 - q _ { 3 } } \left[\tilde{a}_{2}\left(e_{1}+h_{1} x_{1}\right)+\tilde{b}_{2}\left(e_{2}+h_{2} y_{1}\right)+\tilde{c}_{2}\left(e_{3}+h_{3} z_{1}\right)\right.\right.\right. \\
& \left.\left.\left.-h_{3} \tilde{b}_{1} z_{1}-\left(D_{t}^{1-q_{3}} e_{3}(t)\right) \frac{(t)^{-\left(q_{3}-1\right)-1}}{\Gamma\left(-\left(q_{3}-1\right)\right)}-e_{3}\right]\right)+\left(D_{t}^{q_{3}} e_{3}(t)\right) \frac{(t)^{-\left(q_{3}-1\right)-1}}{\Gamma\left(-\left(q_{3}-1\right)\right)}\right] \\
& \left.+\tilde{\mathrm{a}}_{1} \dot{\tilde{\mathrm{a}}}_{1}+\tilde{\mathrm{d}}_{1} \dot{\tilde{\mathrm{d}}}_{1}+\tilde{\mathrm{c}}_{1} \dot{\tilde{\mathrm{c}}}_{1}+\tilde{\mathrm{b}}_{1} \dot{\tilde{\mathrm{b}}}_{1}+\tilde{\mathrm{a}}_{2} \dot{\tilde{\mathrm{a}}}_{2}+\tilde{\mathrm{b}}_{2} \dot{\tilde{\mathrm{b}}}_{2}+\tilde{\mathrm{c}}_{2} \dot{\tilde{\mathrm{c}}}_{2}\right) .
\end{aligned}
$$

Now, using Lemma 2.1, Eq. (3.9) reduces to

$$
\begin{aligned}
\dot{V}= & e_{1}\left[h_{1} \tilde{a}_{1}\left(y_{1}-x_{1}\right)-e_{1}\right]+e_{2}\left[h_{2} \tilde{d}_{1} x_{1}+h_{2} \tilde{c}_{1} y_{1}-e_{2}\right]+e_{3}\left[\tilde{a}_{2}\left(e_{1}+h_{2} x_{1}\right)\right. \\
& \left.+\tilde{b}_{2}\left(e_{2}+h_{2} y_{1}\right)+\tilde{c}_{2}\left(e_{3}+h_{3} z_{1}\right)-h_{3} \tilde{b}_{1} z_{1}-e_{3}\right]+\tilde{a}_{1}\left(-h_{1}\left(y_{1}-x_{1}\right) e_{1}\right) \\
& +\tilde{\mathrm{d}}_{1}\left(-h_{2} x_{1} e_{2}\right)+\tilde{c}_{1}\left(-h_{2} y_{1} e_{2}\right)+\tilde{b}_{1}\left(h_{3} z_{1} e_{3}\right)+\tilde{a}_{2}\left(-\left(e_{1}+h_{1} x_{1}\right) e_{3}\right) \\
& +\tilde{b}_{2}\left(-\left(e_{2}+h_{2} y_{1}\right) e_{3}\right)+\tilde{c}_{2}\left(-\left(e_{3}+h_{3} z_{1}\right) e_{3}\right), \\
= & -e_{1}^{2}-e_{2}^{2}-e_{3}^{2}<0 .
\end{aligned}
$$

Since $\mathrm{V}$ is positive definite and $\dot{V}$ is negative definite in the neighborhood of zero solution of system equation (3.6), it follows $\lim _{t \rightarrow \infty}\|e(t)\|=0$. Therefore system (3.2) can synchronize system (3.1) asymptotically. This completes the proof. 
(a)

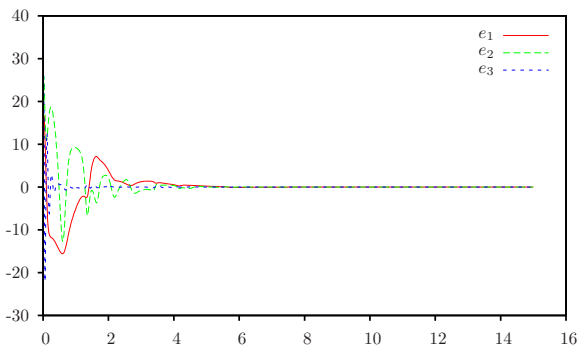

(b)

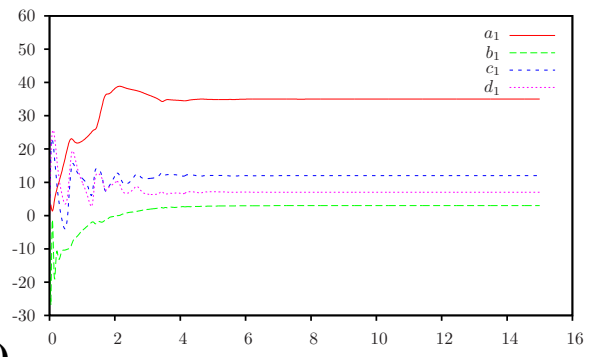

(c)

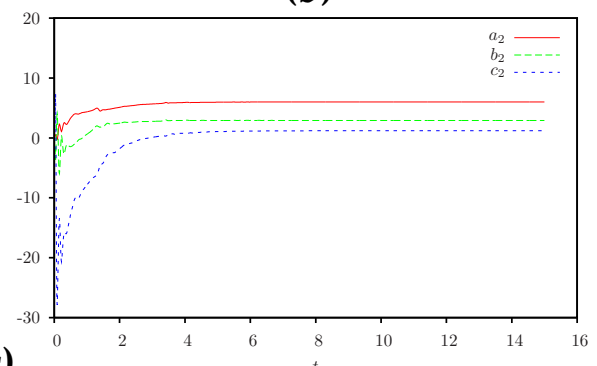

Figure 1: (a): Error signals between drive and response systems, (b)-(c): Estimated values for unknown parameters with same scaling factors in $(x y z)$ projection.

(a)

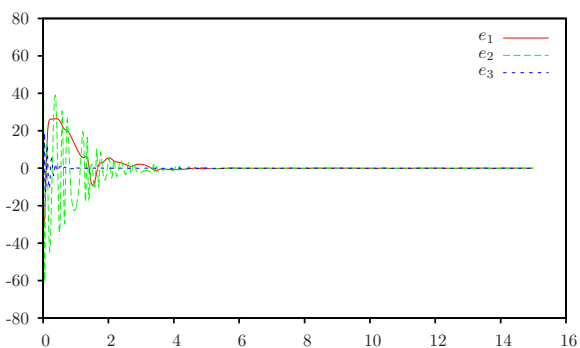

(b)
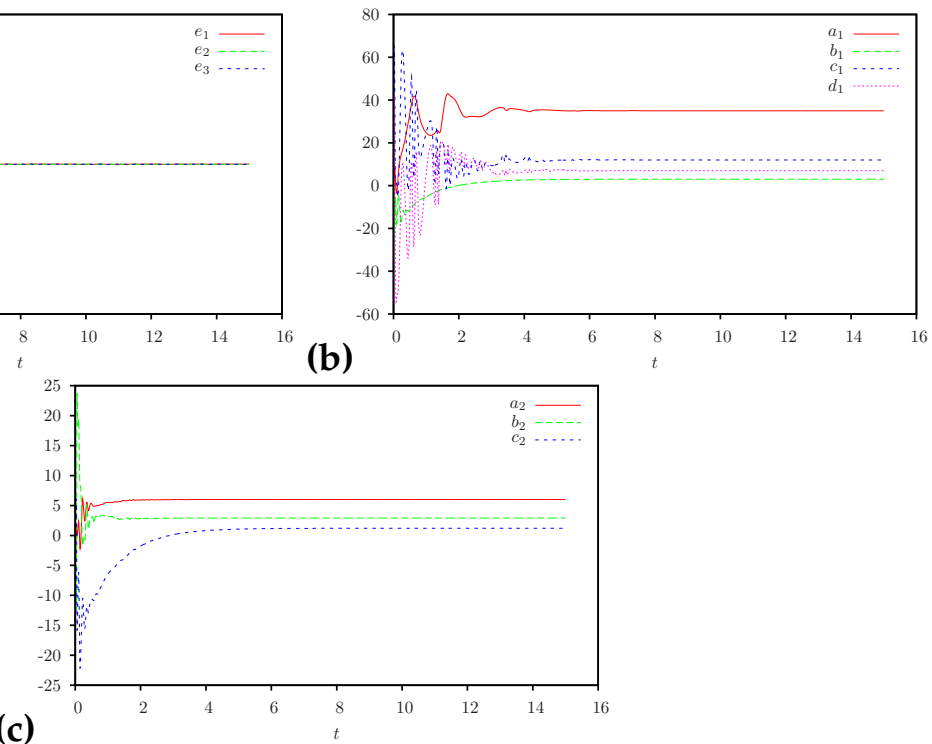

Figure 2: (a): Error signals between drive and response systems, (b)-(c): Estimated values for unknown parameters with different scaling factors in $(x y z)$ projection.

To verify and demonstrate the effectiveness and the feasibility of the presented projective reduce order synchronization method, the simulation results have been performed. In the numerical simulation, the Adams-Bashforth-Moulton method is used to solve the systems. The fractional order is chosen as $\mathrm{q}_{i}=0.94, i=1,2,3,4$ to ensure the hyperchaotic and chaotic behavior. The parameters are always chosen as $\mathrm{a}_{1}=35, \mathrm{~d}_{1}=7, \mathrm{c}_{1}=12, \mathrm{~b}_{1}=3, \mathrm{a}_{2}=6, \mathrm{c}_{2}=1.2, \mathrm{~b}_{2}=2.92$. The initial conditions are $\left(x_{1}(0)=12, y_{1}(0)=22, z_{1}(0)=31, w_{1}(0)=4\right)$ and $\left(x_{2}(0)=0.3, y_{2}(0)=0.7, z_{2}(0)=1.2\right)$. In addition, the initial condition of the parameter update law is $\left(a_{1}(0)=0.2, d_{1}(0)=0.2, c_{1}(0)=0.2, b_{1}(0)=0.2\right)$ and $\left(a_{2}(0)=10, b_{2}(0)=0.2, c_{2}(0)=0.2\right)$. Fig. 1 displays the time evolutions of the dynamics errors and depicts the dynamics of the parameters estimation of the systems (3.1) and (3.2) with the scaling factor 
$h_{i}=-1, i=1,2,3$. Fig. 2 displays the time evolutions of the dynamics errors and depicts the dynamics of the parameters estimation of the systems (3.1) and (3.2) with different scaling factors $h_{1}=2, h_{2}=-3, h_{3}=$ 1. Obviously, the projective reduce order synchronization errors converge asymptotically to zero and two systems (3.1) and (3.2) are indeed achieved with projective reduce order synchronization. Furthermore, the estimated values of unknown converge to $a_{1}=35, d_{1}=7, c_{1}=12, b_{1}=3, a_{2}=6, c_{2}=1.2, b_{2}=2.92$ as $t \rightarrow \infty$.

\section{Projective reduce order synchronization between the projection $(x y w)$ of the fractional order hy- perchaotic Chen and Genesio-Tesi systems}

In order to investigate the projective reduce order synchronization behavior between the projection (xyw) of the fractional order hyperchaotic Chen Eqs. (3.1) and the fractional order Genesio-Tesi systems Eqs. (3.2), we assume that the (xyw) projection of the fractional order hyperchaotic Chen systems is the master system, therefore, the error system can be presented in the form of

$$
\begin{aligned}
D_{t}^{q_{1}} e_{1}(t)= & \left(e_{2}+h_{2} y_{1}\right)-h_{1} a_{1}\left(y_{1}-x_{1}\right)-h_{1} w_{1}+u_{1}, \\
D_{t}^{q_{2}} e_{2}(t)= & \left(e_{3}+h_{3} z_{1}\right)-h_{2} d_{1} x_{1}+h_{2} x_{1} z_{1}-h_{2} c_{1} y_{1}+u_{2}, \\
D_{t}^{q_{3}} e_{3}(t)= & -a_{2}\left(e_{1}+h_{1} x_{1}\right)-b_{2}\left(e_{2}+h_{2} y_{1}\right)-c_{2}\left(e_{3}+h_{3} z_{1}\right)+\left(e_{1}+h_{1} x_{1}\right)^{2} \\
& -h_{3} y_{1} z_{1}-h_{3} r_{1} w_{1}+u_{3},
\end{aligned}
$$

where $e_{1}=x_{2}-h_{1} x_{1}, e_{2}=y_{2}-h_{2} y_{1}, e_{3}=z_{2}-h_{3} w_{1}$ and $h=\operatorname{dig}\left(h_{1}, h_{2}, h_{3}\right)$ is the scaling matrix. Our goal is to derive the controller $\mathrm{U}$ with a parameter estimation update law such that Eqs. (3.1) synchronize Eqs. (3.2), the controllers are taken as

$$
\begin{aligned}
u_{1}= & -\left(e_{2}+h_{2} y_{1}\right)+h_{1} a_{1}\left(y_{1}-x_{1}\right)+h_{1} w_{1}+D_{t}^{q_{1}-1}\left[h_{1} \hat{a}_{1}\left(y_{1}-x_{1}\right)\right. \\
& \left.-\left(D_{t}^{q_{1}-1} e_{1}(t)\right) \frac{(t)-\left(q_{1}-1\right)-1}{\Gamma\left(-\left(q_{1}-1\right)\right)}-e_{1}\right], \\
u_{2}= & -\left(e_{3}+h_{3} z_{1}\right)+h_{2} d_{1} x_{1}-h_{2} x_{1} z_{1}+h_{2} c_{1} y_{1}+D_{t}^{q_{2}-1}\left[h_{1} \hat{d}_{1} x_{1}+h_{2} \hat{c}_{1} y_{1}\right. \\
& \left.-\left(D_{t}^{q_{2}-1} e_{2}(t)\right) \frac{(t)-\left(q_{2}-1\right)-1}{\Gamma\left(-\left(q_{2}-1\right)\right)}-e_{2}\right], \\
u_{3}= & a_{2}\left(e_{1}+h_{1} x_{1}\right)+b_{2}\left(e_{2}+h_{2} y_{1}\right)+c_{2}\left(e_{3}+h_{3} z_{1}\right)-\left(e_{1}+h_{1} x_{1}\right)^{2}+h_{3} y_{1} z_{1} \\
& +h_{3} r_{1} w_{1}+D_{t}^{q_{3}-1}\left[\hat{a}_{2}\left(e_{1}+h_{2} x_{1}\right)+\hat{b}_{2}\left(e_{2}+h_{2} y_{1}\right)+\hat{c}_{2}\left(e_{3}+h_{3} z_{1}\right)+h_{3} \hat{r}_{1} w_{1}\right. \\
& \left.-\left(D_{t}^{q_{3}-1} e_{3}(t)\right) \frac{(t)-\left(q_{3}-1\right)-1}{\Gamma\left(-\left(q_{3}-1\right)\right)}-e_{3}\right],
\end{aligned}
$$

and parameter update rules are

$$
\begin{aligned}
& \dot{\hat{\mathrm{a}}}_{1}=-\mathrm{h}_{1}\left(\mathrm{y}_{1}-\mathrm{x}_{1}\right) e_{1}, \\
& \dot{\hat{\mathrm{d}}}_{1}=-\mathrm{h}_{2} \mathrm{x}_{1} \mathrm{e}_{2}, \\
& \dot{\hat{\mathrm{c}}}_{1}=-\mathrm{h}_{2} \mathrm{y}_{1} \mathrm{e}_{2}, \\
& \dot{\hat{\mathrm{r}}}_{1}=-\mathrm{h}_{3} \mathrm{w}_{1} \mathrm{e}_{3}, \\
& \dot{\hat{\mathrm{a}}}_{2}=-\left(\mathrm{e}_{1}+\mathrm{h}_{1} \mathrm{x}_{1}\right) e_{3}, \\
& \dot{\hat{\mathrm{b}}}_{2}=-\left(e_{2}+\mathrm{h}_{2} \mathrm{y}_{1}\right) e_{3}, \\
& \dot{\hat{\mathrm{c}}}_{2}=-\left(e_{3}+\mathrm{h}_{3} z_{1}\right) e_{3},
\end{aligned}
$$

where, $\hat{a}_{1}, \hat{d}_{1}, \hat{c}_{1}, \hat{r}_{1}, \hat{a}_{2}, \hat{b}_{2}, \hat{c}_{2}$ are estimates of $a_{1}, d_{1}, c_{1}, r_{1}, a_{2}, b_{2}, c_{2}$, respectively.

Theorem 4.1. For given synchronization scaling matrix $h=\operatorname{diag}\left(h_{1}, h_{2}, \ldots, h_{n}\right)$ and any initial conditions, projective reduce order synchronization between system (3.1) and system (3.2) will occur by the control law (4.2) and the updating law of parameters (4.3). 
Proof. Inserting (4.2) into (4.1) yields the following:

$$
\begin{aligned}
D_{t}^{q_{1}} e_{1}(t)= & D_{t}^{q_{1}-1}\left[h_{1} \tilde{a}_{1}\left(y_{1}-x_{1}\right)-\left(D_{t}^{q_{1}-1} e_{1}(t)\right) \frac{(t)^{-\left(q_{1}-1\right)-1}}{\Gamma\left(-\left(q_{1}-1\right)\right)}-e_{1}\right], \\
D_{t}^{q_{2}} e_{2}(t)= & D_{t}^{q_{2}-1}\left[h_{2} \tilde{d}_{1} x_{1}+h_{2} \tilde{c}_{1} y_{1}-\left(D_{t}^{q_{2}-1} e_{2}(t)\right) \frac{(t)^{-\left(q_{2}-1\right)-1}}{\Gamma\left(-\left(q_{2}-1\right)\right)}-e_{2}\right], \\
D_{t}^{q_{3}} e_{3}(t)= & D_{t}^{q_{3}-1}\left[\tilde{a}_{2}\left(e_{1}+h_{1} x_{1}\right)+\tilde{b}_{2}\left(e_{2}+h_{2} y_{1}\right)+\tilde{c}_{2}\left(e_{3}+h_{3} z_{1}\right)+h_{3} \tilde{r}_{1} w_{1}\right. \\
& \left.-\left(D_{t}^{q_{3}-1} e_{3}(t)\right) \frac{(t)^{-\left(q_{3}-1\right)-1}}{\Gamma\left(-\left(q_{3}-1\right)\right)}-e_{3}\right],
\end{aligned}
$$

where $\tilde{\mathrm{a}}_{1}=\hat{\mathrm{a}}_{1}-\mathrm{a}_{1}, \tilde{\mathrm{d}}_{1}=\hat{\mathrm{d}}_{1}-\mathrm{d}_{1}, \tilde{\mathrm{c}}_{1}=\hat{\mathrm{c}}_{1}-\mathrm{c}_{1}, \tilde{\mathrm{r}}_{1}=\hat{\mathrm{r}}_{1}-\mathrm{r}_{1}, \tilde{\mathrm{a}}_{2}=\hat{\mathrm{a}}_{2}-\mathrm{a}_{2}, \tilde{\mathrm{b}}_{2}=\hat{\mathrm{b}}_{2}-\mathrm{b}_{2}, \tilde{\mathrm{c}}_{2}=\hat{\mathrm{c}}_{2}-\mathrm{c}_{2}$. Consider the following Lyapunov function candidate:

$$
\mathrm{V}=\frac{1}{2}\left(e^{\top} e+\tilde{\mathrm{a}}_{1}^{2}+\tilde{\mathrm{d}}_{1}^{2}+\tilde{\mathrm{c}}_{1}^{2}+\tilde{\mathrm{r}}_{1}^{2}+\tilde{\mathrm{a}}_{2}^{2}+\tilde{\mathrm{b}}_{2}^{2}+\tilde{\mathrm{c}}_{2}^{2}\right),
$$

The time derivative of $\mathrm{V}$ along the solution of error dynamical system equation (3.7) gives

$$
\dot{V}=\left(e_{1} \dot{e}_{1}+e_{2} \dot{e}_{2}+e_{3} \dot{e}_{3}+\tilde{\mathrm{a}}_{1} \dot{\tilde{\mathrm{a}}}_{1}+\tilde{\mathrm{d}}_{1} \dot{\tilde{\mathrm{d}}}_{1}+\tilde{\mathrm{c}}_{1} \dot{\tilde{\mathrm{c}}}_{1}+\tilde{\mathrm{r}}_{1} \dot{\tilde{\mathrm{r}}}_{1}+\tilde{\mathrm{a}}_{2} \dot{\tilde{\mathrm{a}}}_{2}+\tilde{\mathrm{b}}_{2} \dot{\tilde{\mathrm{b}}}_{2}+\tilde{\mathrm{c}}_{2} \dot{\tilde{\mathrm{c}}}_{2}\right) \text {. }
$$

Using Lemma 2.2 in Eq. (4.5) we get

$$
\begin{aligned}
& \dot{V}=\left(e_{1}\left[D_{t}^{1-q_{1}}\left(D_{t}^{q_{1}} e_{1}(t)\right)+\left(D_{t}^{q_{1}} e_{1}(t)\right) \frac{(t)^{-\left(q_{1}-1\right)-1}}{\Gamma\left(-\left(q_{1}-1\right)\right)}\right]\right. \\
& +e_{2}\left[D_{t}^{1-q_{2}}\left(D_{t}^{q_{2}} e_{2}(t)\right)+\left(D_{t}^{q_{2}} e_{2}(t)\right) \frac{(t)^{-\left(q_{2}-1\right)-1}}{\Gamma\left(-\left(q_{2}-1\right)\right)}\right] \\
& +e_{3}\left[D_{t}^{1-q_{3}}\left(D_{t}^{q_{3}} e_{3}(t)\right)+\left(D_{t}^{q_{3}} e_{3}(t)\right) \times \frac{(t)^{-\left(q_{3}-1\right)-1}}{\Gamma\left(-\left(q_{3}-1\right)\right)}\right] \\
& \left.+\tilde{\mathrm{a}}_{1} \dot{\tilde{\mathrm{a}}}_{1}+\tilde{\mathrm{d}}_{1} \dot{\tilde{\mathrm{d}}}_{1}+\tilde{\mathrm{c}}_{1} \dot{\tilde{\mathrm{c}}}_{1}+\tilde{\mathrm{r}}_{1} \dot{\tilde{\mathrm{r}}}_{1}+\tilde{\mathrm{a}}_{2} \dot{\tilde{\mathrm{a}}}_{2}+\tilde{\mathrm{b}}_{2} \dot{\tilde{\mathrm{b}}}_{2}+\tilde{\mathrm{c}}_{2} \dot{\tilde{\mathrm{c}}}_{2}\right), \\
& =\left(e _ { 1 } \left[D_{t}^{1-q_{1}}\left(D_{t}^{q_{1}-1}\left[h_{1} \tilde{a}_{1}\left(y_{1}-x_{1}\right)-\left(D_{t}^{q_{1}-1} e_{1}(t)\right) \frac{(t)^{-\left(q_{1}-1\right)-1}}{\Gamma\left(-\left(q_{1}-1\right)\right)}-e_{1}\right]\right)\right.\right. \\
& \left.+\left(D_{t}^{q_{1}} e_{1}(t)\right) \frac{(t)^{-\left(q_{1}-1\right)-1}}{\Gamma\left(-\left(q_{1}-1\right)\right)}\right] \\
& +e_{2}\left[D_{t}^{1-q_{2}}\left(D_{t}^{q_{2}-1}\left[h_{2} \tilde{d}_{1} x_{1}+h_{2} \tilde{c}_{1} y_{1}-\left(D_{t}^{q_{2}-1} e_{2}(t)\right) \frac{(t)^{-\left(q_{2}-1\right)-1}}{\Gamma\left(-\left(q_{2}-1\right)\right)}-e_{2}\right]\right)\right. \\
& \left.+\left(D_{t}^{q_{2}} e_{2}(t)\right) \frac{(t)^{-\left(q_{2}-1\right)-1}}{\Gamma\left(-\left(q_{2}-1\right)\right)}\right] \\
& +e_{3}\left[D _ { t } ^ { 1 - q _ { 3 } } \left(D _ { t } ^ { 1 - q _ { 3 } } \left[\tilde{a}_{2}\left(e_{1}+h_{1} x_{1}\right)+\tilde{b}_{2}\left(e_{2}+h_{2} y_{1}\right)+\tilde{c}_{2}\left(e_{3}+h_{3} z_{1}\right)\right.\right.\right. \\
& \left.\left.\left.+h_{3} \tilde{r}_{1} w_{1}-\left(D_{t}^{1-q_{3}} e_{3}(t)\right) \frac{(t)^{-\left(q_{3}-1\right)-1}}{\Gamma\left(-\left(q_{3}-1\right)\right)}-e_{3}\right]\right)+\left(D_{t}^{q_{3}} e_{3}(t)\right) \frac{(t)^{-\left(q_{3}-1\right)-1}}{\Gamma\left(-\left(q_{3}-1\right)\right)}\right] \\
& \left.+\tilde{\mathrm{a}}_{1} \dot{\tilde{\mathrm{a}}}_{1}+\tilde{\mathrm{d}}_{1} \dot{\tilde{\mathrm{d}}}_{1}+\tilde{\mathrm{c}}_{1} \dot{\tilde{\mathrm{c}}}_{1}+\tilde{\mathrm{r}}_{1} \dot{\tilde{\mathrm{r}}}_{1}+\tilde{\mathrm{a}}_{2} \dot{\tilde{\mathrm{a}}}_{2}+\tilde{\mathrm{b}}_{2} \dot{\tilde{\mathrm{b}}}_{2}+\tilde{\mathrm{c}}_{2} \dot{\tilde{\mathrm{c}}}_{2}\right) .
\end{aligned}
$$

Now, using Lemma 2.1, Eq. (3.9) reduces to

$$
\dot{\mathrm{V}}=e_{1}\left[h_{1} \tilde{\mathrm{a}}_{1}\left(\mathrm{y}_{1}-\mathrm{x}_{1}\right)-e_{1}\right]+e_{2}\left[\mathrm{~h}_{2} \tilde{\mathrm{d}}_{1} \mathrm{x}_{1}+\mathrm{h}_{2} \tilde{\mathrm{c}}_{1} \mathrm{y}_{1}-\mathrm{e}_{2}\right]+\mathrm{e}_{3}\left[\tilde{\mathrm{a}}_{2}\left(\mathrm{e}_{1}+\mathrm{h}_{2} \mathrm{x}_{1}\right)\right.
$$




$$
\begin{aligned}
& \left.+\tilde{b}_{2}\left(e_{2}+h_{2} y_{1}\right)+\tilde{c}_{2}\left(e_{3}+h_{3} z_{1}\right)+h_{3} \tilde{r}_{1} w_{1}-e_{3}\right]+\tilde{a}_{1}\left(-h_{1}\left(y_{1}-x_{1}\right) e_{1}\right) \\
& +\tilde{d}_{1}\left(-h_{2} x_{1} e_{2}\right)+\tilde{c}_{1}\left(-h_{2} y_{1} e_{2}\right)+\tilde{r}_{1}\left(-h_{3} w_{1} e_{3}\right)+\tilde{a}_{2}\left(-\left(e_{1}+h_{1} x_{1}\right) e_{3}\right) \\
& +\tilde{b}_{2}\left(-\left(e_{2}+h_{2} y_{1}\right) e_{3}\right)+\tilde{c}_{2}\left(-\left(e_{3}+h_{3} z_{1}\right) e_{3}\right) \\
& =-e_{1}^{2}-e_{2}^{2}-e_{3}^{2}<0 .
\end{aligned}
$$

Since $\mathrm{V}$ is positive definite and $\dot{V}$ is negative definite in the neighborhood of zero solution of system equation (4.4), it follows $\lim _{t \rightarrow \infty}\|e(t)\|=0$. Therefore system (3.2) can synchronize system (3.1) asymptotically. This completes the proof.

(a)

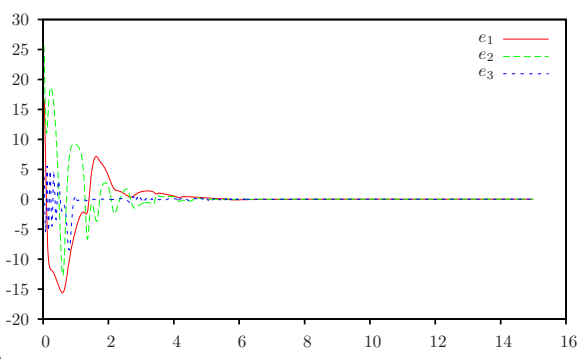

(b)
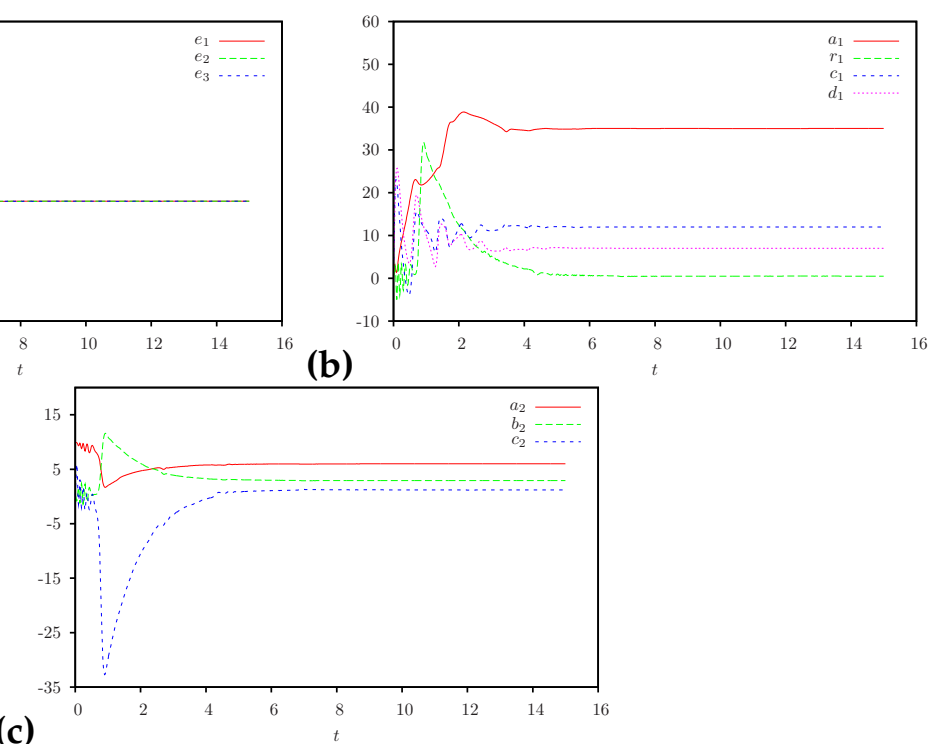

Figure 3: (a): Error signals between drive and response systems, (b)-(c): Estimated values for unknown parameters with same scaling factors in $(x y w)$ projection.

(a)

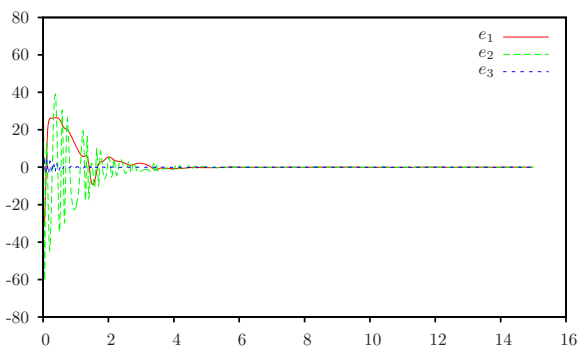

(b)
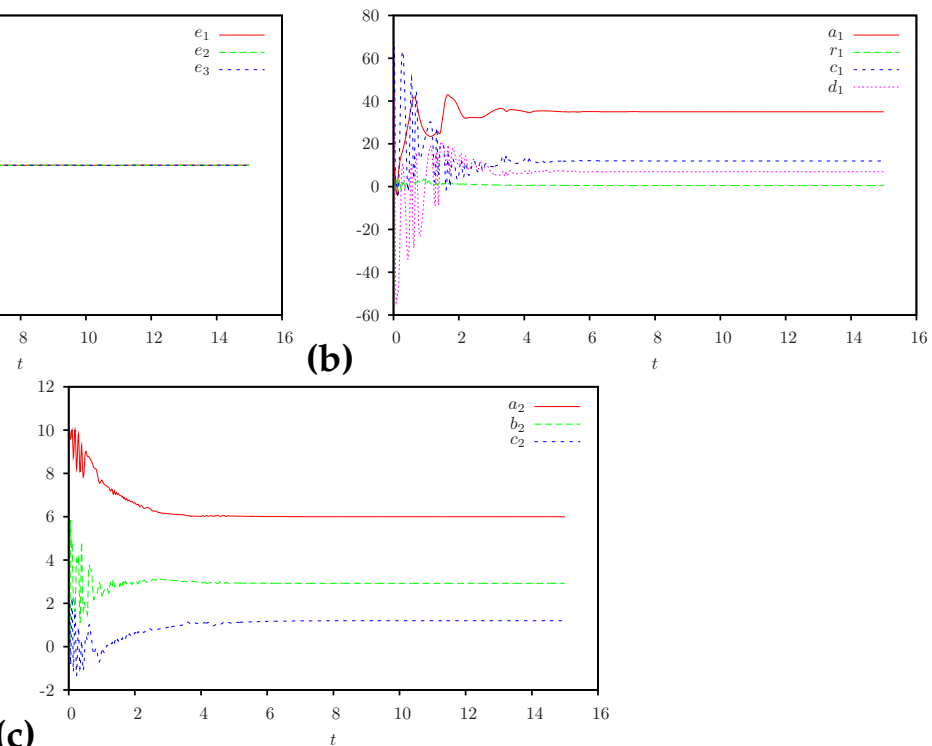

Figure 4: (a): Error signals between drive and response systems, (b)-(c): Estimated values for unknown parameters with different scaling factors in $(x y w)$ projection. 
To verify and demonstrate the effectiveness and the feasibility of the presented projective reduce order synchronization method, the simulation results have been performed. In the numerical simulation, the Adams-Bashforth-Moulton method is used to solve the systems. The fractional order is chosen as $\mathrm{q}_{\mathrm{i}}=0.94, \mathrm{i}=1,2,3,4$ to ensure the hyperchaotic and chaotic behavior. The parameters are always chosen as $a_{1}=35, d_{1}=7, c_{1}=12, r_{1}=0.5, a_{2}=6, c_{2}=1.2, b_{2}=2.92$. The initial conditions are $\left(x_{1}(0)=12, y_{1}(0)=22, z_{1}(0)=31, w_{1}(0)=4\right)$ and $\left(x_{2}(0)=0.3, y_{2}(0)=0.7, z_{2}(0)=1.2\right)$. In addition, the initial condition of the parameter update law is $\left(a_{1}(0)=0.2, d_{1}(0)=0.2, c_{1}(0)=0.2, r_{1}(0)=0.2\right)$ and $\left(a_{2}(0)=10, b_{2}(0)=0.2, c_{2}(0)=0.2\right)$. Fig. 3 displays the time evolutions of the dynamics errors and depicts the dynamics of the parameters estimation of the systems (3.1) and (3.2) with the scaling factor $h_{i}=-1, i=1,2,3$. Fig. 4 displays the time evolutions of the dynamics errors and depicts the dynamics of the parameters estimation of the systems (3.1) and (3.2) with different scaling factors $h_{1}=2, h_{2}=-3, h_{3}=$ 1. Obviously, the projective reduce order synchronization errors converge asymptotically to zero and two systems (3.1) and (3.2) are indeed achieved with projective reduce order synchronization. Furthermore, the estimated values of unknown converge to $a_{1}=35, d_{1}=7, c_{1}=12, r_{1}=0.5, a_{2}=6, c_{2}=1.2, b_{2}=2.92$ as $t \rightarrow \infty$.

\section{Conclusion}

We have studied the projective reduce order synchronization of uncertain chaotic systems with different order. A novel adaptive reduce order synchronization controllers with corresponding parameter update laws is proposed to projective synchronize the projection of the fourth-order fractional order hyperchaotic Chen system with the third order fractional order chaotic Genesio-Tesi system even though their parameters are unknown. This technique has been successfully applied to several examples. Theoretical analysis via the Lyapunov stability theory and numerical simulations have been shown to verify our results for the projective reduce order synchronization of the proposed method.

\section{Acknowledgment}

The author thanks the Deanship of Research at the University of Hail, Saudi Arabia, for funding this work under Grant no. 0150353. Also the author is grateful to the anonymous referees for their useful comments on the earlier draft of this paper.

\section{References}

[1] S. K. Agrawal, S. Das, A modified adaptive control method for synchronization of some fractional chaotic systems with unknown parameters, Nonlinear Dynam., 73 (2013), 907-919. 1, 2.1, 2.2, 2.3

[2] S. K. Agrawal, S. Das, Function projective synchronization between four dimensional chaotic systems with uncertain parameters using modified adaptive control method, J. Proc. Control, 24 (2014), 517-530. 1, 2.1, 2.2, 2.3

[3] S. K. Agrawal, M. Srivastava, S. Das, Synchronization of fractional order chaotic systems using active control method, Chaos Solitons Fractals, 45 (2012), 737-752. 1

[4] I. Ahmad, A. B. Saaban, A. B. Ibrahim, M. Shahzad, N. Naveed, The synchronization of chaotic systems with different dimensions by a robust generalized active control, Optik, 127 (2016), 4859-4871. 1

[5] M. M. Al-sawalha, A. Al-Sawalha, Anti-synchronization of fractional order chaotic and hyperchaotic systems with fully unknown parameters using modified adaptive control, Open Phys., 14 (2016), 304-313. 1

[6] M. M. Al-sawalha, M. S. M. Noorani, Chaos reduced-order anti-synchronization of chaotic systems with fully unknown parameters, Commun. Nonlinear Sci. Numer. Simul., 17 (2012), 1908-1920. 1

[7] D. Baleanu, G.-C. Wu, Y.-R. Bai, F.-L. Chen, Stability analysis of Caputolike discrete fractional systems, Commun. Nonlinear Sci. Numer. Simul., 48 (2017), 520-530. 1

[8] S. Bhalekar, V. Daftardar-Gejji, Synchronization of different fractional order chaotic systems using active control, Commun. Nonlinear Sci. Numer. Simul., 15 (2010), 3536-3546. 1

[9] A. Bouzeriba, A. Boulkroune, T. Bouden, Projective synchronization of two different fractional-order chaotic systems via adaptive fuzzy control, Neural Comput. Appl., 27 (2016), 1349-1360. 1

[10] L.-P. Chen, S.-B. Wei, Y. Chai, R.-C. Wu, Adaptive projective synchronization between two different fractional-order chaotic systems with fully unknown parameters, Math. Probl. Eng., 2012 (2012), 16 pages. 1, 2.1 
[11] M. R. Faieghi, H. Delavari, Chaos in fractional-order Genesio-Tesi system and its synchronization, Commun. Nonlinear Sci. Numer. Simul., 17 (2012), 731-741. 3

[12] Z. Gao, X.-Z. Liao, Integral sliding mode control for fractional-order systems with mismatched uncertainties, Nonlinear Dynam., 72 (2013), 27-35. 1

[13] A. K. Golmankhaneh, R. Arefi, D. Baleanu, Synchronization in a nonidentical fractional order of a proposed modified system, J. Vib. Control, 21 (2015), 1154-1161. 1

[14] A. Hajipour, S. S. Aminabadi, Synchronization of chaotic Arneodo system of incommensurate fractional order with unknown parameters using adaptive method, Optik, 127 (2016), 7704-7709. 1

[15] R. Hilfer (Ed.), Applications of fractional calculus in physics, World Scientific Publishing Co., Inc., River Edge, NJ, (2001). 1

[16] W. Jawaada, M. S. M. Noorani, M. M. Al-Sawalha, Anti-synchronization of chaotic systems via adaptive sliding mode control, Chin. Phys. Lett., 29 (2012), 1-3. 1

[17] R. Mainieri, J. Rehacek, Projective synchronization in three-dimensional chaotic systems, Phys. Rev. Lett., 82 (1999), 3042-3045. 1, 2.1

[18] L. M. Pecora, T. L. Carroll, Synchronization in chaotic systems, Phys. Rev. Lett., 64 (1990), 821-824. 1

[19] I. Podlubny, Fractional differential equations, An introduction to fractional derivatives, fractional differential equations, to methods of their solution and some of their applications, Mathematics in Science and Engineering, Academic Press, Inc., San Diego, CA, (1999). 1, 2.1, 2.2, 2.3

[20] A. G. Radwan, K. Moaddy, K. N. Salama, S. Momani, I. Hashim, Control and switching synchronization of fractional order chaotic systems using active control technique, J. Adv. Res., 5 (2014), 125-132. 1

[21] X.-N. Song, S. Song, B. Li, Adaptive synchronization of two time-delayed fractional-order chaotic systems with different structure and different order, Optik, 127 (2016), 11860-11870. 1

[22] Z. Wang, X. Huang, H. Shend, Control of an uncertain fractional order economic system via adaptive sliding mode, Neurocomputing, 83 (2012), 83-88. 1

[23] Q. Wang, D.-L. Qi, Synchronization for fractional order chaotic systems with uncertain parameters, Int. J. Control Autom. Syst., 14 (2016), 211-216. 1

[24] S. Wang, Y. Yu, M. Diao, Hybrid projective synchronization of chaotic fractional order systems with different dimensions, Phys. A, 389 (2010), 4981-4988. 1, 2.1

[25] G.-C. Wu, D. Baleanu, Chaos synchronization of the discrete fractional logistic map, Signal Process., 102 (2014), 96-99. 1

[26] G.-C. Wu, D. Baleanu, Discrete fractional logistic map and its chaos, Nonlinear Dynam., 75 (2014), 283-287. 1

[27] G.-C. Wu, D. Baleanu, S.-D. Zeng, Discrete chaos in fractional sine and standard maps, Phys. Lett. A, 378 (2014), 484-487. 1

[28] X.-J. Wu, Y. Lu, Generalized projective synchronization of the fractional-order Chen hyperchaotic system, Nonlinear Dynam., 57 (2009), 25-35. 3

[29] X.-J. Wu, H.-T. Lu, S.-L. Shen, Synchronization of a new fractional-order hyperchaotic system, Phys. Lett. A, 373 (2009), 2329-2337. 1

[30] Y. Xu, H. Wang, D. Liu, H. Huang, Sliding mode control of a class of fractional chaotic systems in the presence of parameter perturbations, J. Vib. Control, 21 (2015), 435-448. 1

[31] X.-J. Yang, J. A. T. Machado, J. Hristov, Nonlinear dynamics for local fractional Burgers' equation arising in fractal flow, Nonlinear Dynam., 84 (2016), 3-7. 1

[32] X.-J. Yang, J. A. T. Machado, H. M. Srivastava, A new numerical technique for solving the local fractional diffusion equation: two-dimensional extended differential transform approach, Appl. Math. Comput., 274 (2016), 143-151. 1

[33] P. Zhou, R.-J. Bai, The adaptive synchronization of fractional-order chaotic system with fractional-order $1<\mathrm{q}<2$ via linear parameter update law, Nonlinear Dynam., 80 (2015), 753-765. 1

[34] M. Zribi, N. Smaoui, and H. Salim, Synchronization of the unified chaotic systems using a sliding mode controller, Chaos Solitons Fractals, 42 (2009) 3197-3209. 1 